\title{
EFEITO DE PRODUTOS ALTERNATIVOS PARA O CONTROLE DO BOLOR VERDE (Penicillium digitatum) EM PÓS-COLHEITA DE CITROS ${ }^{1}$
}

\author{
DANIEL ANDRADE DE SIQUEIRA FRANCO² \& WAGNER BETTIOL ${ }^{3}$
}

\begin{abstract}
RESUMO - O objetivo do trabalho foi verificar a ocorrência de sinergismo entre misturas de produtos alternativos aos fungicidas, para o controle do bolor verde (Penicillium digitatum) em pós-colheita de citros. Foram testados dez produtos individualmente e trinta e cinco combinações destes produtos dois a dois, em comparação com tiabendazole e testemunha, com e sem inoculação. Os produtos testados não apresentaram efeito de sinergismo, exceto a mistura carbonato de $\mathrm{Na}+$ ácido bórico. Carbonato de Na e ácido bórico controlaram a doença em 78 e $87 \%$, respectivamente, e, utilizando a mistura, o controle foi de $93 \%$. Destacaram-se, ainda no controle da doença, o bicarbonato de $\mathrm{Na}$, metabissulfito de $\mathrm{Na}$ e as misturas de bicarbonato de sódio + ácido bórico, carbonato de $\mathrm{Na}+$ carbonato de K, carbonato de $\mathrm{Na}+$ sorbato de $\mathrm{K}$, bicarbonato de $\mathrm{Na}$ + carbonato de $\mathrm{Na}$, controlando 92; 77; 81; 77; 75 e $71 \%$, respectivamente. $\mathrm{O}$ tiabendazole utilizado como padrão controlou totalmente a doença.
\end{abstract}

Termos para indexação: conservadores de alimentos, controle alternativo, citrus.

\section{EFFECT OF THE ALTERNATIVE PRODUCTS FOR CONTROL OF GREEN MOLD (Penicillium digitatum) IN POST-HARVEST CITRUS FRUIT}

\begin{abstract}
The objective of this work was verify the ocurrence of synergism of mixtures for alternative products to the fungicides for the control of the green mold (Penicillium digitatum) in post-harvest citrus fruits. The efficiency of ten products, tested individually, and thirty five combinations among them, in pairs, were compared to thiabendazole and control, with and without inoculation. The products didn't present a synergism effect, except the mixture sodium carbonate + boric acid, that had a disease control of $93 \%$. The products, sodium carbonate and boric acid controled 78 and $87 \%$, respectively. The sodium bicarbonate, sodium methabisulphite and the mixtures of sodium bicarbonate + boric acid, sodium carbonate + potassium carbonate, sodium carbonate + potassium sorbate, sodium bicarbonate + sodium carbonate had a disease control of 92\%,77\%,81\%,77\%, 75\% and 71\%, respectively. The fungicide treatment with thiabendazole used by standard had a whole disease control.
\end{abstract}

Index terms: food conservatives, alternative control, citrus.

O bolor verde, causado por Penicillium digitatum, é a principal doença em pós-colheita de frutos cítricos em todo o mundo. Como forma de controlar esse patógeno, vêm sendo utilizadas práticas culturais visando a reduzir o inóculo no campo, o tratamento químico, a irradiação e a termoterapia. Os tratamentos químicos são os mais utilizados, em pré e pós-colheita. No Brasil, os fungicidas do grupo dos benzimidazóis são os mais utilizados, e estes possuem várias restrições de uso, como o de selecionar estirpes resistentes dos patógenos quando usados continuamente. Alternativas visando à redução do uso de fungicidas vêm sendo pesquisadas e com resultados promissores no controle de vários fitopatógenos. Enfoque particular vem sendo dado ao controle biológico e ao uso de extratos de planta, de produtos alimentares, de aditivos de alimentos, de resíduos da produção de alimentos e de conservadores de alimentos no controle de doenças de plantas de forma geral (Sholberg \& Gaunce, 1995).

Vários sais orgânicos e inorgânicos, alguns dos quais são usados no processamento de alimentos na indústria, apresentam propriedades antimicrobianas e podem ser usados em tratamentos pós-colheita de citros, para o controle do bolor verde (Smilanick et al., 1997; Smilanick et al., 1999; Franco \& Bettiol, 1999). Eles têm sido testados para a inibição de patógenos fúngicos em culturas no campo, em plantas ornamentais e em frutos na pós-colheita (Horst et al., 1992). Em pós-colheita, geralmente, são utilizados em banhos de imersão ou aplicados juntamente à cera de recobrimento dos frutos (Smilanick et al., 1999).

$\mathrm{Na}$ indústria de alimentos, dois ou mais conservadores químicos são freqüentemente encontrados para agirem sinergicamente, sendo mais efetivos do que tratamentos individuais no controle do crescimento microbiano. Dessa forma, o presente trabalho teve como objetivo testar o potencial da combinação de soluções de conservadores de alimentos e de extratos de plantas, para o controle de P. digitatum em frutos de laranja 'Pêra' em pós-colheita.

A inibição da germinação dos conídios de $P$. digitatum

1 (Trabalho 264/2000). Recebido: 04/12/2000. Aceito para publicação: 04/06/2002.

2 Engo Agrọ ${ }^{\circ}$ M.Sc, Aluno do curso de pós-graduação, Proteção de Plantas, FCA/UNESP/Botucatu, C.P. 237, CEP 18603-970, Botucatu-SP, Bolsista do CNPq.

3 Eng ${ }^{\mathrm{o}}$ Agr ${ }^{-}$, pesquisador da EMBRAPA Meio Ambiente, C.P. 69, CEP 13.820-000, Jaguariúna-SP, Bolsista do CNPq. 
foi realizada pelo método do flavedo (Franco \& Bettiol, 2000), empregando-se as soluções dos produtos individualmente. A porcentagem de inibição da germinação de conídios de $P$. digitatum com tiabendazole a $0,15 \%$, bicarbonato de $\mathrm{Na}$ a $3 \%$ e carbonato de $\mathrm{Na}$ a $1 \%$ foram de 18; 95 e $96 \%$, respectivamente. Para o metabissulfito de $\mathrm{Na}$ a $1 \%$, o sorbato de $\mathrm{K}$ a $1 \%$, o carbonato de $\mathrm{K}$ a $1 \%$ e o ácido bórico a $1 \%$ na inibição da germinação dos conídios foi de $100 \%$ (Tabela 1).

Os ensaios foram realizados com frutos no estádio fisiológico maduro, classificados quanto ao tamanho por meio de equipamento e selecionados manualmente. Posteriormente, os frutos foram lavados em solução de hipoclorito de sódio a $0,5 \%$ (v/v) por três minutos e enxaguados duas vezes em água de torneira. Em seguida, os frutos foram feridos com um tubo vazado ( $3 \mathrm{~mm}$ de diâmetro), em dois pontos opostos na região equatorial, a uma profundidade de $\pm 2 \mathrm{~mm}$, atingindo a região do albedo. Após o ferimento, os frutos foram inoculados com 20 $\mathrm{mL}$ de uma suspensão de conídios de $P$. digitatum $\left(1,7 \times 10^{6}\right.$ conídios. $\mathrm{mL}^{-1}$ ). Em seguida, em cada ferimento, foram aplicados $20 \mathrm{~mL}$ da suspensão dos produtos alternativos a serem testados (Kavanagh \& Wood, 1971). Todos os produtos foram comparados com as testemunhas, com e sem inoculação do patógeno, e com o fungicida tiabendazole a $0,15 \%$ (Tabela 2). Após a inoculação, os frutos foram incubados à temperatura de $25 \pm 5^{\circ} \mathrm{C}$, umidade relativa de $85-90 \%$ e fotoperíodo de $12 / 12$ horas. Transcorridos cinco dias, foram realizadas avaliações da incidência e da severidade da doença. A porcentagem de controle da doença foi calculada a partir do diâmetro médio das lesões de cada tratamento, em relação ao controle inoculado. O delineamento experimental foi o inteiramente casualizado, com dez frutos em cada tratamento, com dois ferimentos opostos em cada fruto, totalizando vinte repetições por tratamento.

Os produtos alternativos promissores, carbonato de $\mathrm{Na}$ $1 \%$ + ácido bórico $1 \%$, bicarbonato de $\mathrm{Na} 3 \%$, ácido bórico $1 \%$, bicarbonato de $\mathrm{Na} 3 \%$ + ácido bórico 1\%, carbonato de sódio $1 \%$, carbonato de $\mathrm{Na} 1 \%$ + carbonato de $\mathrm{K} 1 \%$, metabissulfito de $\mathrm{Na} 1 \%$, carbonato de sódio $1 \%$ + sorbato de $\mathrm{K} 1 \%$ e bicarbonato de $\mathrm{Na} 3 \%$ + carbonato de $\mathrm{Na} 1 \%$, controlaram a doença em 93,0;
92,$1 ; 87,7 ; 81,7 ; 78,5 ; 77,6 ; 77,0 ; 75,5$ e $71,6 \%$, respectivamente, quando comparados ao controle com tiabendazole, que teve eficiência de controle de $100 \%$ (Tabela 2). Entretanto, não houve diferença estatisticamente significativa entre os tratamentos quando considerada a severidade da doença. Os demais tratamentos apresentaram controle da doença inferiores a 70\%. Considerando-se ambos os produtos constituintes de cada mistura, apresentou incremento no controle da doença apenas o tratamento de carbonato de $\mathrm{Na} 1 \%$ + ácido bórico $1 \%$ (sendo 14,5\% maior em relação ao tratamento de carbonato de $\mathrm{Na}$ e 5,3\% em relação ao ácido bórico, individualmente). Os demais tratamentos, constituídos de mistura de produtos alternativos, não apresentaram efeito de sinergismo (Tabela 2).

Smilanick et al. (1999) observaram que a imersão de frutas cítricas em soluções de bicarbonato e carbonato de sódio reduziu a incidência do bolor verde em pós-colheita causado por $P$. digitatum, resultados semelhantes aos obtidos no presente trabalho (Tabela 2). Este tratamento pode ser ainda uma ferramenta útil no manejo de isolados resistentes aos fungicidas (Bus et al., 1991). A eficiência desses sais é semelhante à dos fungicidas empregados para o controle do bolor verde e, em geral, superior aos outros tratamentos que são alternativos aos fungicidas (Smilanick \& Denis-Arrue, 1992). Franco \& Bettiol (1999) relataram que o uso dos conservadores de alimentos é seguro, podendo-se usar os bicarbonatos em todos os produtos agrícolas com isenção de resíduos de tolerância. Também, os carbonatos e bicarbonatos são classificados pela legislação americana, como ingredientes aprovados em produtos rotulados como ‘orgânicos', em regulamentação proposta para padronizar as práticas orgânicas.

Os produtos alternativos promissores podem fazer parte de um manejo para o controle do bolor verde, integrando as técnicas de pós-colheita com as técnicas de pré-colheita. Essa tecnologia é perfeitamente compatível com o processo de manuseio dos frutos cítricos em casas de embalagens. Apresentam, ainda, baixo custo e possibilitam a sua utilização a curto prazo, em vista do crescimento do mercado interno de frutos cítricos in natura.

TABELA 1 - Inibição da germinação de conídios de Penicilium digitatum com produtos alternativos aos fungicidas, empregando o método do flavedo.

\begin{tabular}{|l|c|}
\hline \multicolumn{1}{|c|}{ Produtos } & \% de inibiçãoda germinação* \\
\hline Testemunha (água) & 0 \\
\hline Bicarbonato K 1\% & 0 \\
\hline Alanina 1\% & 0 \\
\hline Tween 80 1,7\% (T) & 0 \\
\hline Cymbopogon cytratus 5\%+ T & 0 \\
\hline Glutamato Na 1\% & 18 \\
\hline Thiabenzazole 0,15\% & 95 \\
\hline Bicarbonato Na 3\% & 96 \\
\hline Carbonato Na 1\% & 100 \\
\hline Metabissulfito Na 1\% & 100 \\
\hline Sorbato K 1\% & 100 \\
\hline Carbonato K 1\% & 100 \\
\hline Ácidobórico 1\% & \\
\hline
\end{tabular}

* Média de nove discos de tecido de flavedo, observação em dez campos por disco, após 21 horas de incubação em câmara úmida e escura a $25 \pm 2^{\circ} \mathrm{C}$. 
TABELA 2 - Controle em pós-colheita do bolor verde, causado por Penicillium digitatum em frutos de laranja 'Pêra', por produtos alternativos.

\begin{tabular}{|c|c|c|c|c|}
\hline Tratamento & Incidência $^{x}$ & Severidade $^{y}$ & & Controle \% \\
\hline Tween 80 a $1,7 \%(\mathrm{~T})$ & 100 & 97,0 & $\mathrm{a}$ & 0,0 \\
\hline Testemunha com inóculo & 100 & 93,5 & a & 0,0 \\
\hline C. citratus $5 \%+\mathrm{T}+$ Glutamato $\mathrm{Na} 1 \%$ & 100 & 88,7 & $a b$ & 5,2 \\
\hline Alanina $1 \%$ & 100 & 83,6 & abc & 10,6 \\
\hline Metabissulfito Na $1 \%+$ Bicarbonato K $1 \%$ & 100 & 80,6 & abcd & 13,9 \\
\hline Bicarbonato $\mathrm{Na} 3 \%+$ Metabissulfito $\mathrm{Na} 1 \%$ & 100 & 75,5 & abcde & 19,3 \\
\hline Glutamato $\mathrm{Na} 1 \%$ & 100 & 74,6 & abcde & 20,2 \\
\hline C. citratus $5 \%+\mathrm{T}+$ Metabissulfito $\mathrm{Na} 1 \%$ & 100 & 74,0 & abcde & 20,8 \\
\hline C. citratus $5 \%+\mathrm{T}+\mathrm{Bicarbonato} \mathrm{K} 1 \%$ & 90 & 70,4 & abcdef & 24,7 \\
\hline Sorbato K $1 \%+$ Bicarbonato K $1 \%$ & 100 & 68,5 & abcdefg & 26,7 \\
\hline Metabissulfito $\mathrm{Na} 1 \%$ + Carbonato K $1 \%$ & 100 & 68,4 & abcdefg & 26,8 \\
\hline Sorbato K $1 \%+$ Metabissulfito Na $1 \%$ & 100 & 67,5 & abcdefgh & 27,8 \\
\hline C. citratus $5 \%+\mathrm{T}+$ Carbonato $\mathrm{Na} 1 \%$ & 100 & 66,9 & abcdefghi & 28,4 \\
\hline C. citratus $5 \%+\mathrm{T}+$ Carbonato $\mathrm{K} 1 \%$ & 100 & 66,0 & abcdefghi & 29,4 \\
\hline Carbonato $\mathrm{Na} 1 \%$ + Metabissulfito $\mathrm{Na} 1 \%$ & 100 & 66,0 & abcdefghi & 29,5 \\
\hline Sorbato K $1 \%+$ Alanina $1 \%$ & 90 & 66,0 & abcdefghi & 29,5 \\
\hline C. citratus $5 \%+\mathrm{T}+$ Sorbato $\mathrm{K} 1 \%$ & 100 & 65,6 & abcdefghi & 29,9 \\
\hline C. citratus $5 \%+\mathrm{T}+$ Alanina $1 \%$ & 100 & 64,7 & abcdefghi & 30,8 \\
\hline Metabissulfito $\mathrm{Na} 1 \%$, Glutamato $\mathrm{Na} 1 \%$ & 100 & 64,6 & abcdefghi & 31,0 \\
\hline C. citratus $5 \%+\mathrm{T}+$ Ácido bórico $1 \%$ & 100 & 55,6 & abcdefghij & 40,6 \\
\hline Metabissulfito Na $1 \%$ +Alanina $1 \%$ & 100 & 51,1 & abcdefghijk & 45,4 \\
\hline Sorbato K $1 \%$ & 100 & 50,8 & abcdefghijk & 45,7 \\
\hline Carbonato $\mathrm{Na} 1 \%$ + Glutamato $\mathrm{Na} 1 \%$ & 90 & 47,7 & abcdefghijk & 48,9 \\
\hline Sorbato K $1 \%$ + Glutamato Na $1 \%$ & 90 & 46,5 & abcdefghijk & 50,2 \\
\hline Sorbato K $1 \%$ + Carbonato K $1 \%$ & 100 & 44,8 & abcdefghijk & 52,1 \\
\hline Carbonato $\mathrm{Na} 1 \%+$ Alanina $1 \%$ & 90 & 43,5 & abcdefghijkl & 53,5 \\
\hline Carbonato K $1 \%$ & 90 & 43,0 & abcdefghijkl & 54,0 \\
\hline C. citratus $5 \%+\mathrm{T}+$ Bicarbonato Na $3 \%$ & 100 & 42,5 & abcdefghijkl & 54,5 \\
\hline Bicarbonato Na 3\% + Sorbato K 1\% & 90 & 41,9 & abcdefghijklm & 55,2 \\
\hline Bicarbonato $\mathrm{K} 1 \%$ & 80 & 41,3 & abcdefghijklm & 55,8 \\
\hline Bicarbonato Na 3\%+ Carbonato K $1 \%$ & 70 & 40,7 & abcdefghijklm & 56,4 \\
\hline Bicarbonato $\mathrm{Na} 3 \%+\mathrm{Bicarbonato} \mathrm{K} 1 \%$ & 80 & 35,2 & bcdefghijklm & 62,3 \\
\hline Bicarbonato $\mathrm{Na} 3 \%$ + Glutamato $\mathrm{Na} 1 \%$ & 70 & 35,2 & bcdefghijklm & 62,3 \\
\hline Sorbato K $1 \%$ + Ácido bórico $1 \%$ & 90 & 35,0 & bcdefghijklm & 62,5 \\
\hline Cymbopogon citratus $5 \%+\mathrm{T}$ & 80 & 31,0 & cdefghijklm & 66,8 \\
\hline Bicarbonato $\mathrm{Na} 3 \%+$ Alanina $1 \%$ & 70 & 30,7 & cdefghijklm & 67,2 \\
\hline Carbonato $\mathrm{Na} 1 \%+$ Bicarbonato K $1 \%$ & 80 & 29,6 & defghijklm & 68,3 \\
\hline Metabissulfito Na $1 \%$ + Ácido bórico $1 \%$ & 80 & 28,1 & efghijklm & 70,0 \\
\hline Bicarbonato $\mathrm{Na} 3 \%$ + Carbonato $\mathrm{Na} 1 \%$ & 70 & 26,6 & efghijklmn & 71,6 \\
\hline Carbonato $\mathrm{Na} 1 \%$ + Sorbato K $1 \%$ & 60 & 23,0 & fghijklm n & 75,5 \\
\hline Metabissulfito Na $1 \%$ & 90 & 21,6 & ghijklmn & 77,0 \\
\hline Carbonato $\mathrm{Na} 1 \%$ + Carbonato K $1 \%$ & 60 & 20,9 & hijklmn & 77,6 \\
\hline Carbonato $\mathrm{Na} 1 \%$ & 50 & 20,1 & ijklmn & 78,5 \\
\hline Bicarbonato $\mathrm{Na} 3 \%$ + Ácido bórico $1 \%$ & 60 & 17,1 & $\mathrm{jklmn}$ & 81,7 \\
\hline Ácido bórico $1 \%$ & 50 & 11,5 & $\mathrm{k} \operatorname{lmn}$ & 87,7 \\
\hline Bicarbonato $\mathrm{Na} 3 \%$ & 40 & 7,4 & $1 \mathrm{mn}$ & 92,1 \\
\hline Carbonato $\mathrm{Na} 1 \%$ + Ácido bórico $1 \%$ & 40 & 6,6 & $\mathrm{mn}$ & 93,0 \\
\hline Tiabendazole $0,15 \%$ & 0 & 0,0 & $\mathrm{n}$ & 100,0 \\
\hline Testemunha sem inóculo $\left(\mathrm{H}_{2} \mathrm{O}\right)$ & 0 & 0,0 & $\mathrm{n}$ & - \\
\hline
\end{tabular}

${ }^{\mathrm{x}}$ Dez frutos por tratamento; ${ }^{y} \mathrm{~S}=\left(\right.$ diâmetro médio da lesão/altura média de todos os frutos do ensaio)x100; Leitura após 5 dias de incubação a $25^{\circ} \mathrm{C} \pm 5$ e $85-$ $90 \%$ UR.

Misturas das soluções dos tratamentos foram realizadas na proporção de 1:1 (v/v). Para efeito de análise estatística, os dados foram transformados em arc sen da raiz quadrada de $\mathrm{x} / 100$.

* Médias seguidas de mesmas letras na coluna não diferem entre si, ao nível de significância de 5\%, pelo teste de Tukey.

\section{REFERÊNCIAS BIBLIOGRÁFICAS}

BUS, V.G., BONGERS, A.J., RISSE, L.A. Occurrence of Penicillium digitatum and Penicillium italicum resistent to benomyl, thiabendazlole, and imazalil on citrus fruit from different geographic origens. Plant Disease, St. Paul, v. 75, p. 1098-1100, 1991.
FRANCO, D.A.S., BETTIOL, W. Controle do Bolor Verde em Pós-Colheita de Citros com Produtos Alternativos. In: AMBROSIANO, E. (Coord.) Agricultura Ecológica. Guaíba: Agropecuária. 1999, p. 121-130.

FRANCO, D.A.S., BETTIOL, W. Eficiência do método do flavedo para avaliar a germinação de conídios de Penicillium digitatum, agente causal do bolor verde dos frutos cítricos. Summa 
Phytopathologica, Jaboticabal, v. 26, n. 2, p. 265-268, 2000.

HORST, R.K., KAWAMOTO, S.O., PORTER, L.L. Effect of sodium bicarbonate and oils on the control of powdery mildew and black spot of roses. Plant Disease, St. Paul, v. 76, p. 247-251, 1992.

KAVANAGH, J.A., WOOD, R.K.S. Green mould of oranges caused by Penicillium digitatum Sacc.; effect of additives on spore germination and infection. Annals of Applied Biology, Wellesbourne, v. 67, p. 35-44, 1971.

SHOLBERG, P.L., GAUNCE, A.P. Fumigation of fruit with acetic acid to prevent postharvest decay. Horticultural Science, Stuttgart, v. 30, p. 1271-1275, 1995.

SMILANICK, J.L., DENIS-ARRUE, R. Control of green mold of lemons with Pseudomonas species. Plant Disease, St. Paul, v. 76, p. 481-485, 1992.
SMILANICK, J.L., MACKEY, B.E., REESE, R., USALL, J., MARGOSAN, D.A. Influence of concentration of soda ash, temperature, and immersion period on the control of postharvest green mold of oranges. Plant Disease, St. Paul, v. 81, p. 379-382, 1997.

SMILANICK, J.L., MARGOSAN, D.A., MLIKOTA, F., USALL, J., MICHAEL, I.F. Control of citrus green mold by carbonate and bicarbonate salts and the influence of comercial postharvest practices on their efficacy. Plant Disease, St. Paul, v. 83, p. 139$145,1999$.

SMILANICK, J.L., MICHAEL, I.F., MANSOUR, M.F., MACKEY, B.E., MARGOSAN, D.A., FLORES, D., WEIST, C.F. Improved control of green mold of citrus with imazalil in warm water compared with its use in wax. Plant Disease, St. Paul, v. 81, n. 11, p. 1299-1304, 1997. 